\title{
Astrometric Observations of Wide Southern Double Stars - II
}

\author{
Christina Bauer ${ }^{\mathrm{A}}$, Graeme L. White ${ }^{\mathrm{A}}$, David L. Blank ${ }^{\mathrm{A}, \mathrm{C}}$, Alex Hons ${ }^{\mathrm{A}}$, \\ and Paul A. Jones ${ }^{\mathrm{B}}$ \\ ${ }^{\text {A }}$ Centre for Astronomy, James Cook University, Townsville QLD 4811, Australia \\ ${ }^{\text {B }}$ School of Physics, University of New South Wales, Sydney NSW 2052, Australia \\ ${ }^{\mathrm{C}}$ Corresponding author. Email: david.blank@jcu.edu.au
}

Received 2007 July 20, accepted 2007 September 5

\begin{abstract}
Astrometric CCD observations have been made of wide ( $\sim 3$ to $60 \operatorname{arcsec})$ southern double stars selected from the Washington Double Star catalogue (WDS). Southern double stars have not been well studied in the past; typically they had not been measured since about 1930, and $\sim 50 \%$ of them have been observed only once before our observations. Of the pairs measured $\sim 80 \%$ show no evidence of motion since the last observation. This is Paper II in which we present the observations of 290 WDS stars in the approximate RA range $17^{\mathrm{h}} 13^{\mathrm{m}}$ to $07^{\mathrm{h}} 30^{\mathrm{m}}$ and in the declination range $-70^{\circ}$ to $-60^{\circ}$. We suggest 412 companions for these 290 stars and list $29(10 \%)$ pairs that have shown significant motion.
\end{abstract}

Keywords: Stars: double, multiple, binary

\section{Introduction}

Double star observations have historically been important in astronomy as the mass of stars is best determined through the astrometry of dynamic binary star systems and the accurate determinations of stellar masses have been used in calibrating the mass-luminosity relationship. The work presented here, however, grew out of our interest in spacecraft astrometry, where double stars had the potential to confuse guidance sensors that work on interferometric principals (see Blackmore et al. 1990; White et al. 1991; Argue et al. 1992; Dommanget 1992). The study of binary stars has a host of other uses, such as probing the conditions of star formation, probing stellar evolution and chemical composition (by identifying pairs of similar age and make up) and others (Chanamé \& Gould 2004). Since accurate observations of double stars give important constraints in stellar modelling, additional data can only help improve the models.

Recent compilations of double star observations in Lampens and Strigachev (2001) and Oblak et al. (1999) have focused on double stars with intermediate (110 arcsec) separations. This paper concentrates on far southern stars with separations greater than $\sim 3$ arcsec.

This paper contains observations of 290 stellar systems lying chiefly between Declination $-60^{\circ}$ to $-70^{\circ}$ and between Right Ascension $17^{\mathrm{h}} 13^{\mathrm{m}}$ to $07^{\mathrm{h}}$ $30^{\mathrm{m}}$ (J2000). This is $\sim 60 \%$ of the neglected zone between $-70^{\circ}$ and $-60^{\circ}$. Pairs were selected from the WDS (Washington Double Star Catalog; http: / / ad. usno. navy. mil/wds/) with catalogued separations of $\sim 3$ arcsec or more to ensure that they could be resolved and accurately measured. There are a small number just outside of this zone as selection was made based on B1950 coordinates. In addition, the region of the Large Magellanic Cloud $\left(04^{\mathrm{h}} 40^{\mathrm{m}}\right.$ to $06^{\mathrm{h}} 00^{\mathrm{m}}$ and south of Declination $-65^{\circ}$ ) was excluded to avoid the considerable confusion due to background stars. The limiting magnitude of the primary component was 13.0, this being the limiting magnitude of the $0.15 \mathrm{~m}$ finder telescope. We summarise below the details concerning the observations and data precision since a fuller account is available in Bauer, White \& Hons (1994; hereafter refereed to as Paper I). We also discuss our methodology for determining which double stars are likely to be physically associated.

\section{Observations and Data Precision}

Observations were made during three periods, 6-10 October 1992, 4-25 November 1992 and 8-12 December 1993, with the last session being primarily concerned with calibration. The mean epoch of the observations is 1992.9. We used an SBIG ST6 CCD camera mounted on the $f / 18$ focus of the 24 inch $(0.6 \mathrm{~m})$ telescope of Mount Stromlo and Siding Spring Observatory (MSSSO) at Siding Spring Observatory (SSO), Australia. Images were obtained of each star through uncalibrated Cousins R and I filters. The stars were sufficiently bright that exposure times could be kept short, typically $180 \mathrm{~s}$ or less.

Raw relative positions and differential magnitudes were determined using the software supplied with the camera (CCDOPS) which does a least-squares fit to the images after subtraction of the sky background. Observations were not made with precise photometry as the principal objective, and indeed were often made on nights that were not photometric. The quoted precision of the magnitude differences represents well the precision of the data. 
Post calibration was undertaken relative to five reference astrometric pairs (Geffert, Sinachopoulos \& Guilbert 1992) to obtain both the image scale and the camera orientation. In addition, the well studied pair $\alpha$ Cen and 'fixed' pairs, $\beta$ PAus and h3670 Ret (Norton's 2000), were also observed to check the consistency of the observations from night-to-night.

The plate scale determined from these observations was found to be $18.13 \pm 0.02$ arcsec per $\mathrm{mm}, 0.9641 \pm 0.0009$ relative to the nominal value for the $f / 18$ focus of the $0.6 \mathrm{~m}$ telescope. The orientation of the camera was determined with an accuracy of $0^{\circ} .04$. There is little evidence for changes in these calibration parameters either throughout the individual nights or from night to night, however, any change in the image scale between observing runs was calibrated out. All measured position angles (PAs) were corrected for the position of the pole at equinox J2000; corrections are typically less than $0^{\circ} .08$. The rms uncertainties in the separation $(\rho)$ and PA are respectively $\Delta \rho=0^{\prime} .16$ and $\Delta \mathrm{PA}=7^{\circ} .8 / \rho(\rho$ in arcsec $)$.

\section{Probability of Physical Association/ Chance Alignment}

In Paper I we concluded that $\sim 18$ percent of the stars listed in this region of the WDS show significant movement since their last measurement which, for the most part, was around 1920 (they ranged from 1850 to 1980 with a median at 1920 ; the majority $(44 \%)$ were made in the period 1910-19; see figure 1a of Paper I).

For the remainder the measured $\rho$ and PA are in good agreement with the corresponding values in the WDS indicating that there has been no significant relative motion between the presented observations and the last prior recorded observation. The comparison between the catalogued WDS figures and our measurements can be found in figures 4 and 5 of Paper I.

We report here an additional 122 stars seen in proximity to WDS primaries. These are fainter stars that appeared in the image, and it remains to be determined whether they are previously undetected faint companions of a multiple system or merely background stars (these are called optical doubles). All of these additional companion stars are (a) closer than 60 arcsec, (b) less than 7.0 magnitudes fainter than the primary in the R and I bands, and are (c) no fainter than $(\mathrm{V}+\Delta \mathrm{R}=$ or $\mathrm{V}+\Delta \mathrm{I}=)$ magnitude 17.0.

In the absence of observable orbital motion or proper motion measurements, the question of whether any two stars are physically related becomes a matter of the probability determined from the magnitudes of the stars, the separation between them and the density of background stars.

It is easily show that the probability of a secondary star of magnitude $m$ being a chance positional alignment with a previously known star is:

$$
\text { Probability }=2.4 \times 10^{-7} N \rho^{2}
$$

where $N$ is the number of stars (per square degree) brighter than magnitude $m$, and $\rho$ is the separation in arcsec.
Note that $N$ depends on the magnitude of the secondary star and the galactic latitude. These values are interpolated from the data in Cox (2000).

In the Notes column of Table 1 we indicate the probability of chance alignment of all stars in Table 1 as a percentage; the column is blank for 274 stars (66\%) as the probability is less than $1 \%$.

Table 1 is the observation of 290 southern wide double stars selected from the WDS. Multiple line entries are for multiple companions of the same WDS pair. We propose 412 stars as companions for these, hoping to define new multiple systems. The table consists of 12 columns. Columns 1, 2 and 3 list the WDS Nomenclature, J2000 Right Ascension and Declination. Columns 4 and 5 give the separation $\rho$ measured from the images taken respectively through the $\mathrm{R}$ and I filters with the average given in column 6. For the 290 WDS primaries and their WDS designated secondaries, the median separation is 11 arcsec (average 16.3 arcsec). Similarly, columns 7, 8 and 9 are the PA for equinox and epoch J2000 measured in R, I and their average.

The $\mathrm{V}$ magnitude of the primary star listed in column 10 is taken from the WDS. Columns 11 and 12 contain the difference in magnitude between the primary and the secondary star measured respectively in R and I. The median value for the 290 WDS primaries and their WDS catalogued secondaries (the pair as listed in the WDS) is 1.6 magnitude in both wavebands (the secondary is typically 1.6 magnitude fainter than the primary). The precision of these data is represented by the significant figures in this column. Negative values apply to stars of very close magnitudes where the original discoverer designated the dimmer star as the primary, or where one of the stars has varied in brightness.

The notes are in column 13. The numerical value is the percentage probability of chance alignment of a background star. No numerical value indicated that the percentage probability is less than $1 \%$ (see above). M ('moved') designates the 29 secondary stars have moved more than 10 arcsec relative to the primary of the period between the first available observation and ours (Table 2).

\section{Stars that Have Moved}

In Paper I we concentrated on comparison of separation and position angle with the last recorded observations recorded in an earlier version of the WDS Catalogue. This was done to establish the reliability of our dataset. Most of the measurements there were made around 1920 and we reported that $\sim 18 \%$ of the stars had moved.

For this paper we have compared our positions with the earliest recorded position in the new format WDS Catalog. These observations were made earlier still, starting in the 1820s. This gives a longer time-line of up to 180 years (typically 120 years), but at the cost of possibly poorer data at the earlier epoch. The majority (45\%) of the early observations were made in a rush of activity in the $1830 \mathrm{~s}$ or between 1890 and $1930(47 \%)$. 


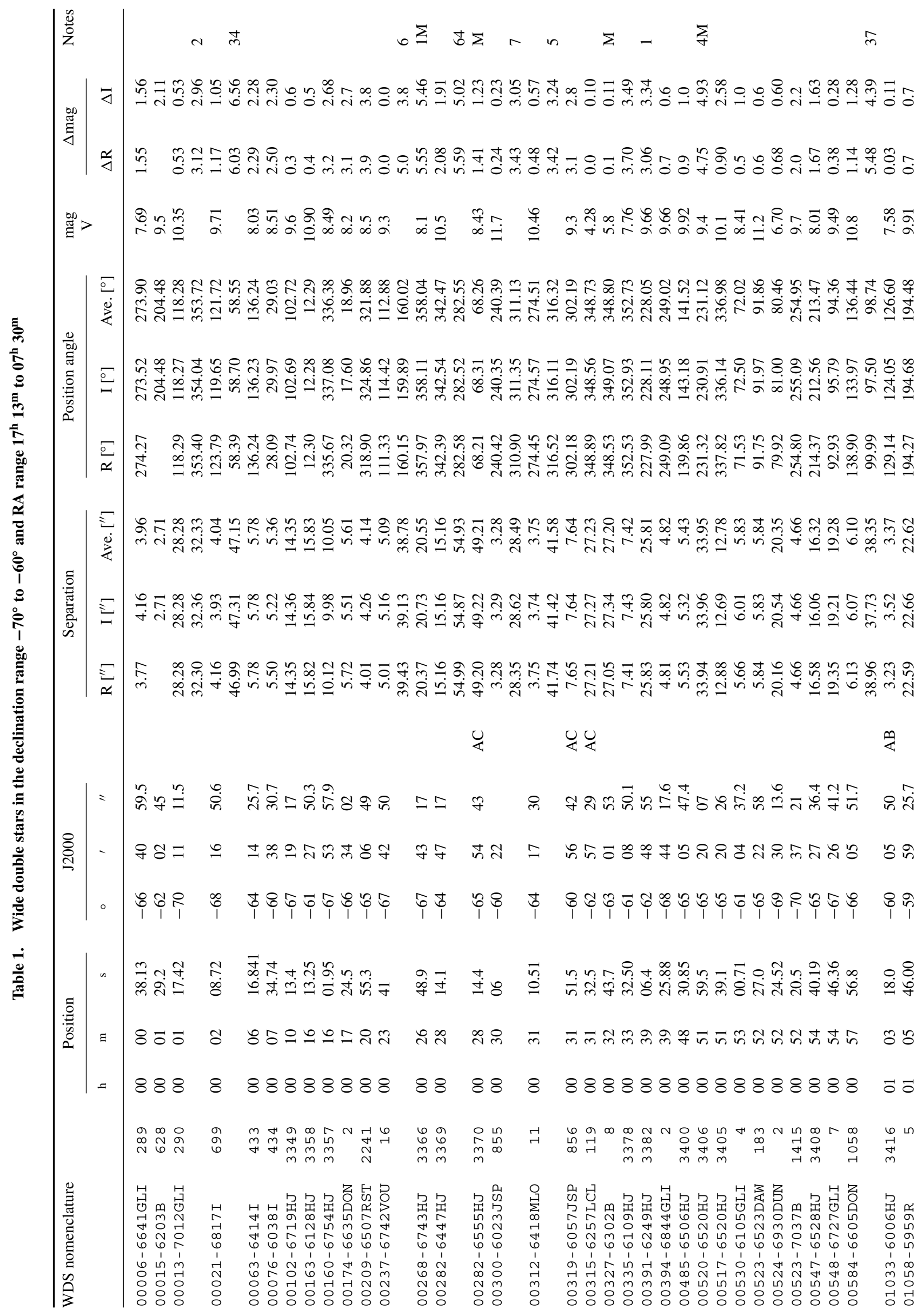




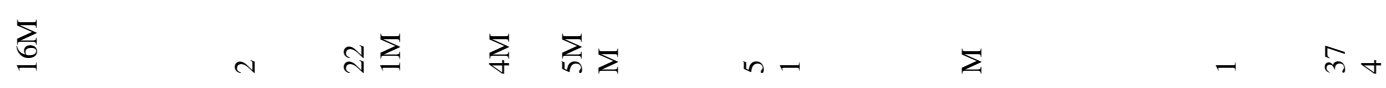

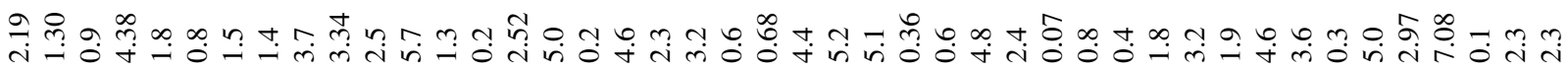

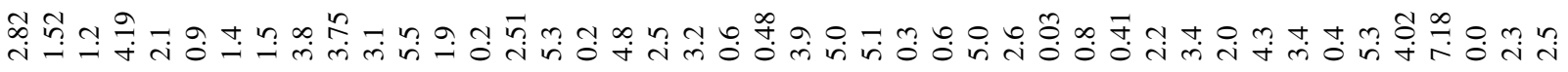

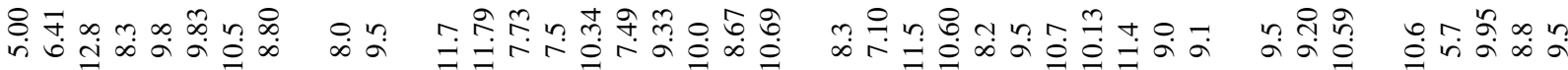

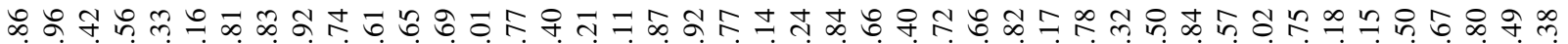

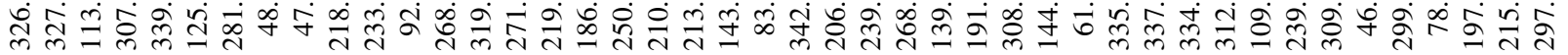

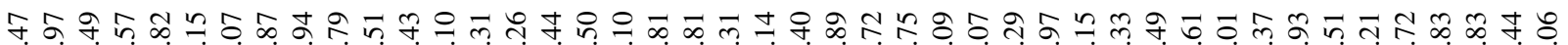

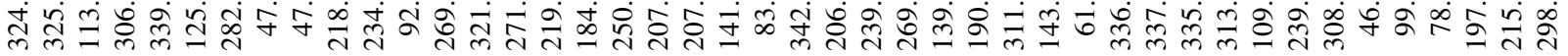

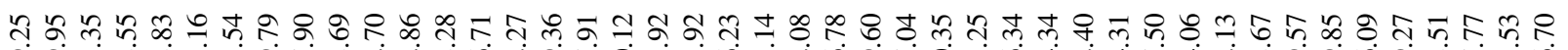

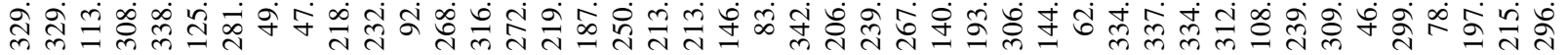

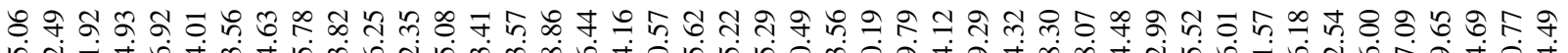

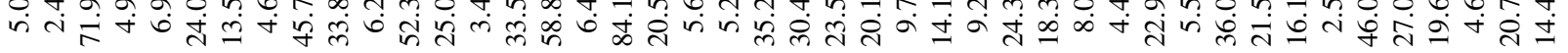

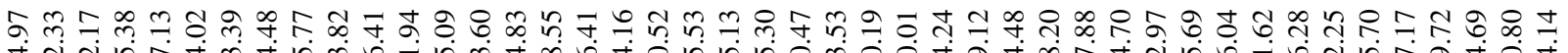
+

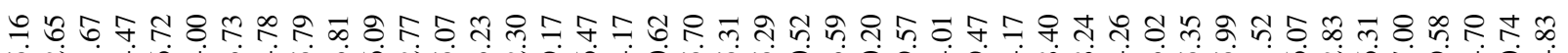
的 तं

$\stackrel{m}{\gtrless}$

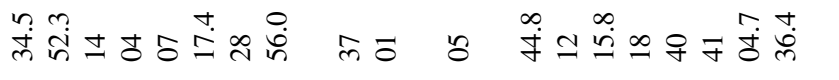

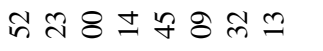

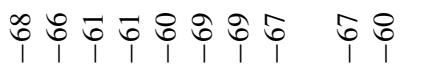

ทำmo+ भुव உニ๙্ণ ๘するするする

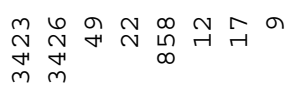

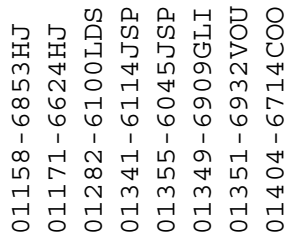

$\stackrel{\circ}{\mathfrak{n}}$

足导 


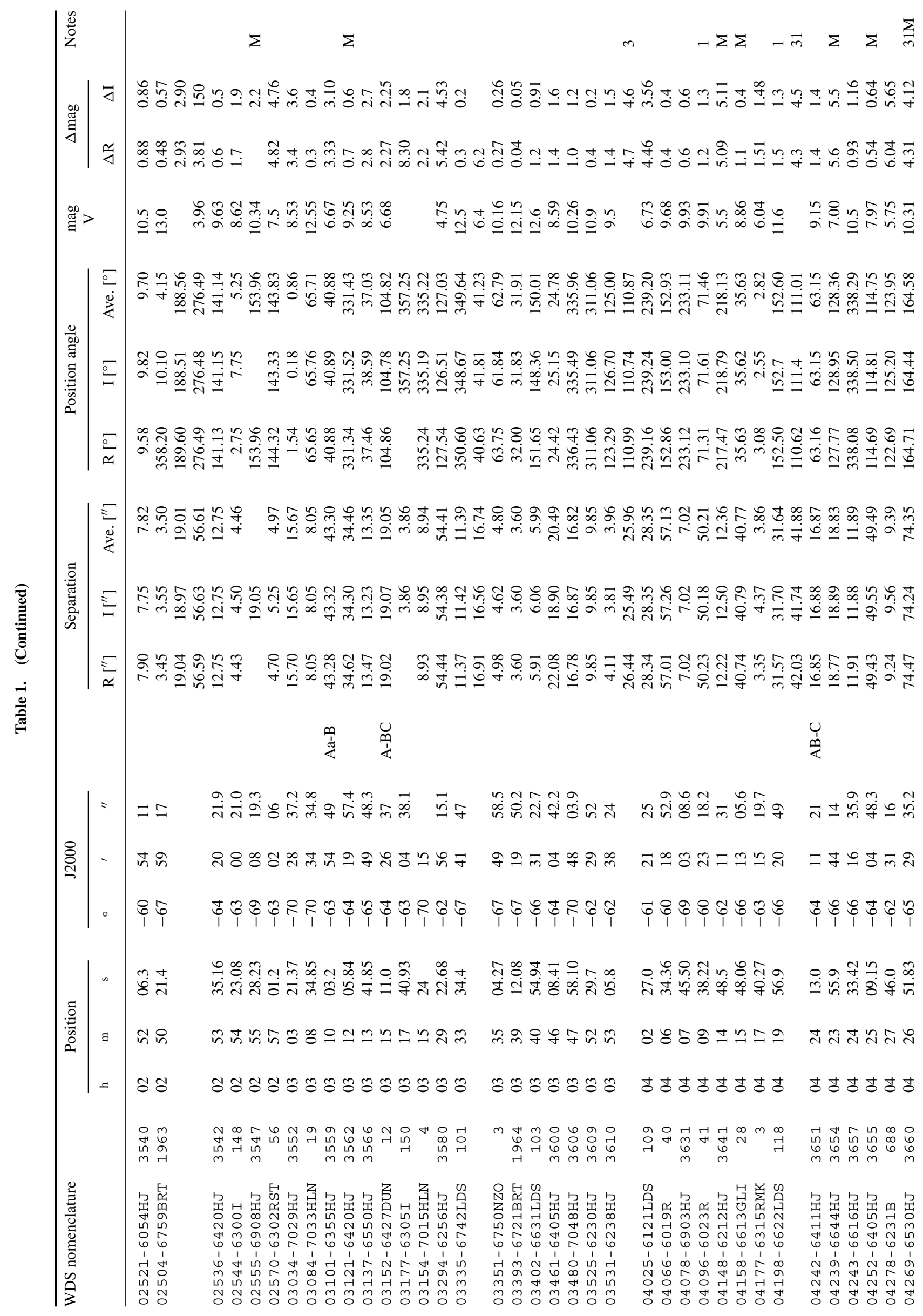




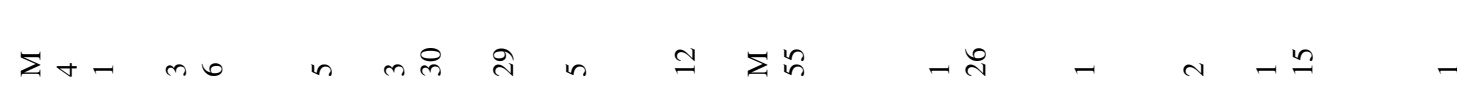

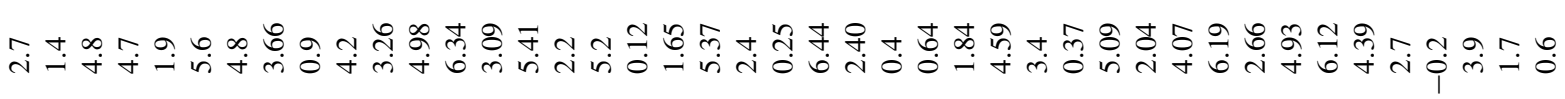

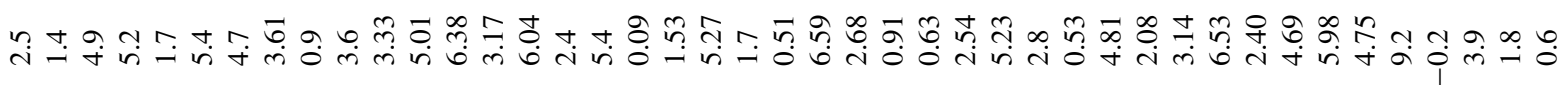

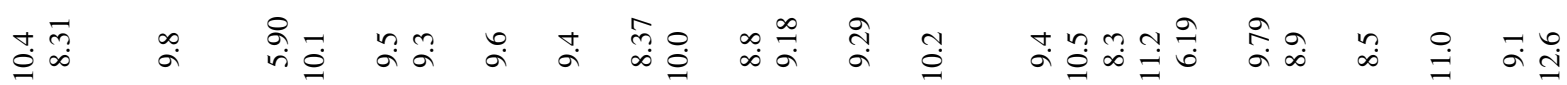

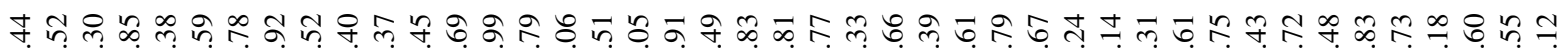

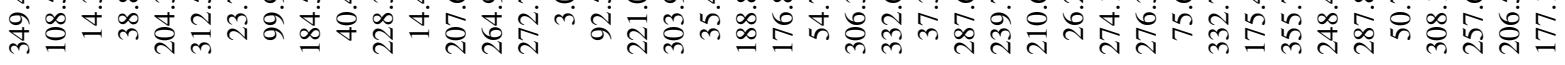

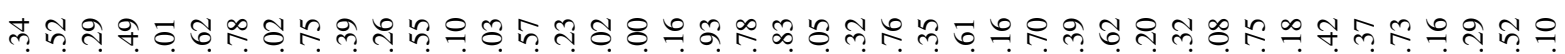

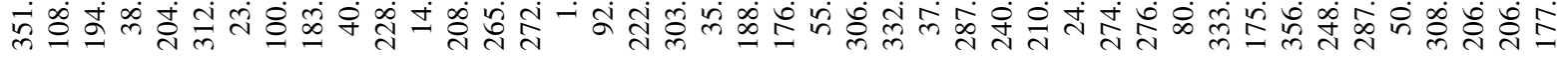

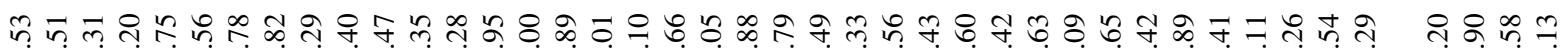

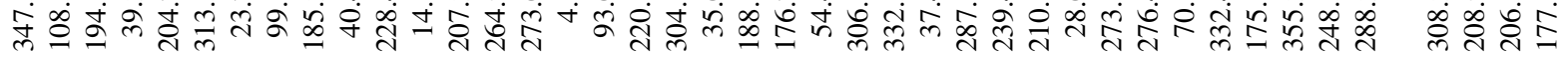

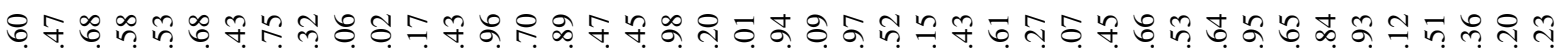

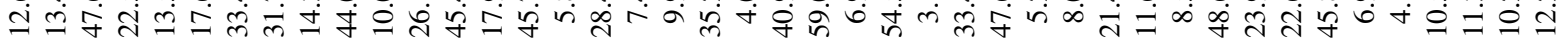

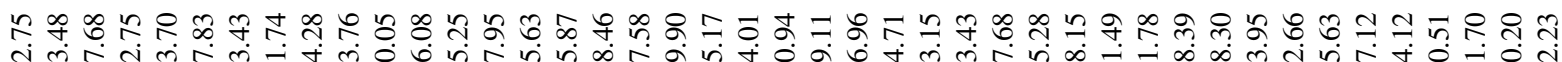

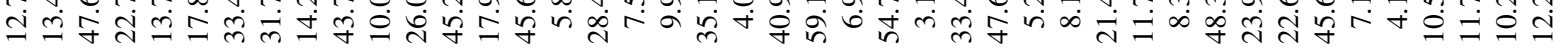

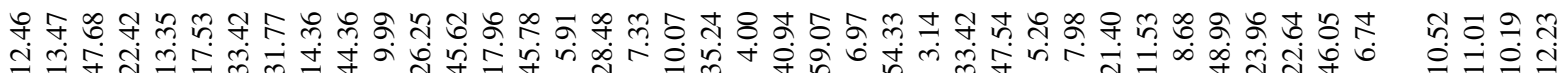
乎这定

8 ๆ ニ ํ ชำ

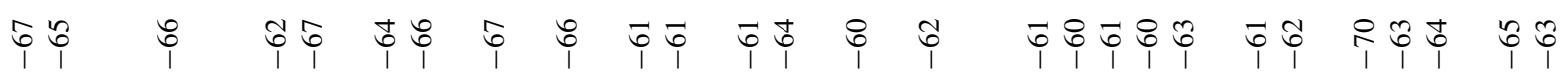

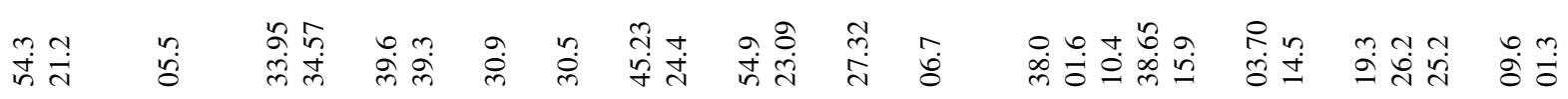

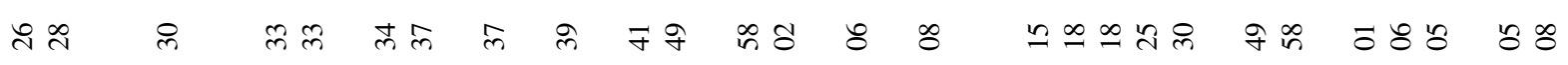
यद

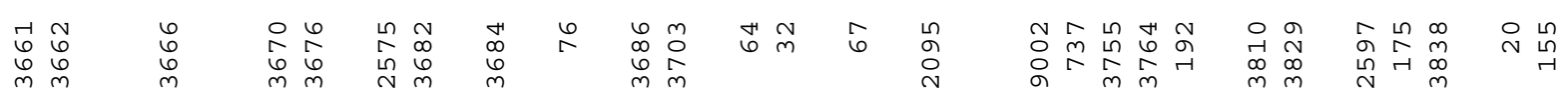

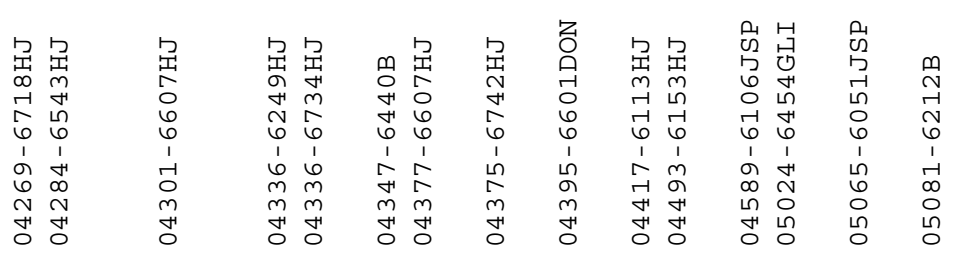

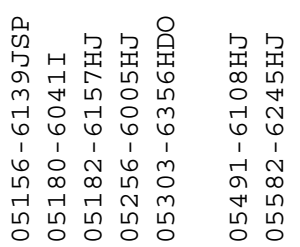

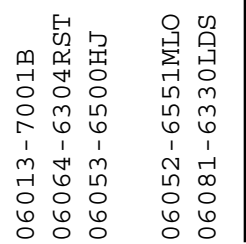




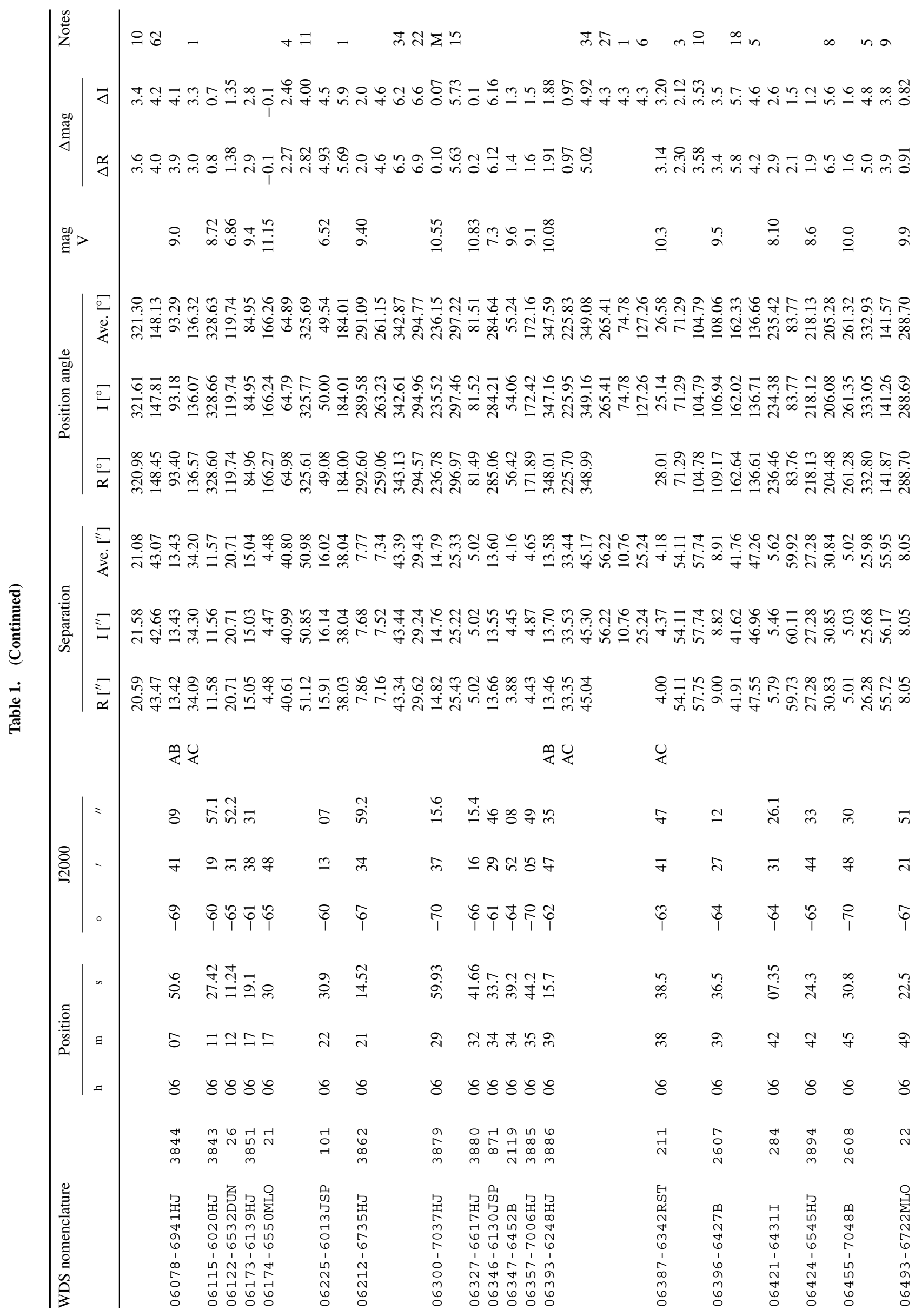




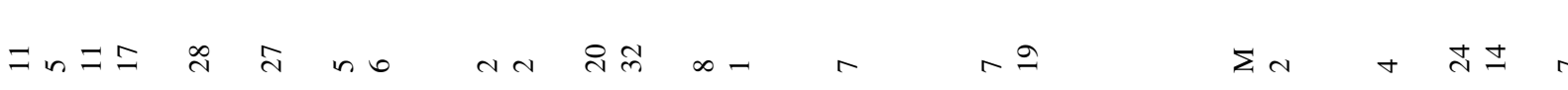

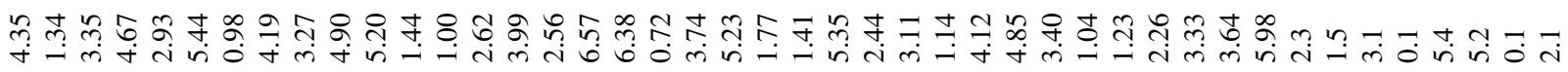

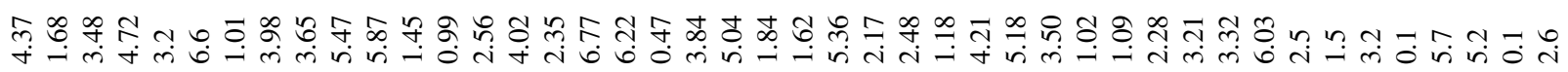

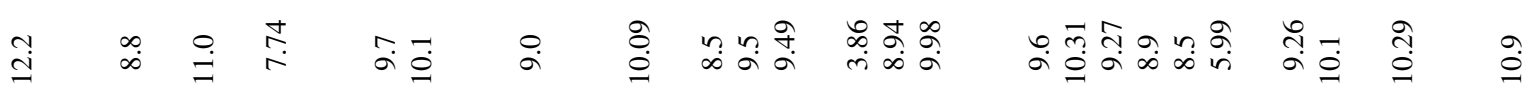

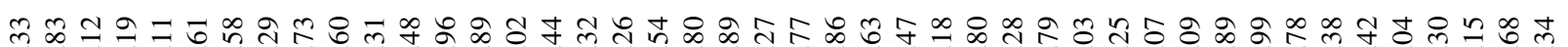
तु m त्ले

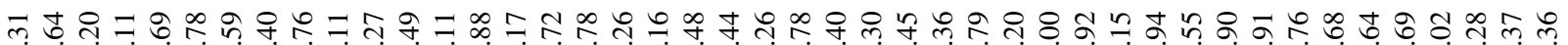

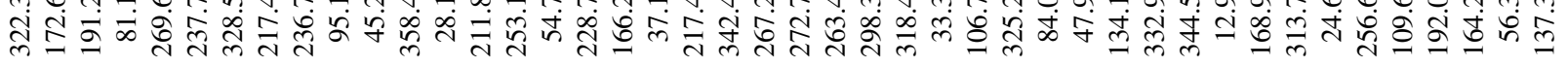
б̆

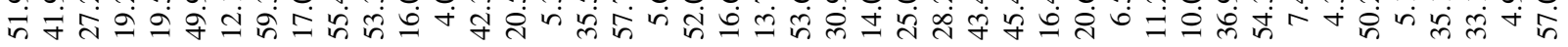

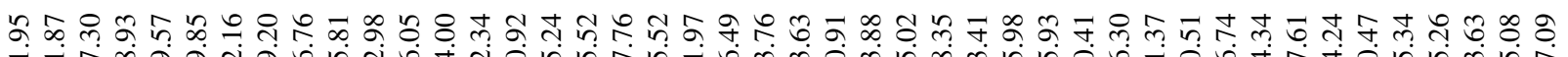

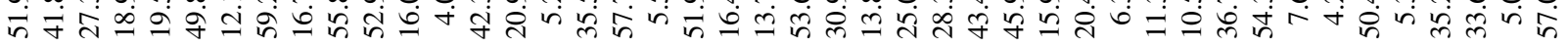

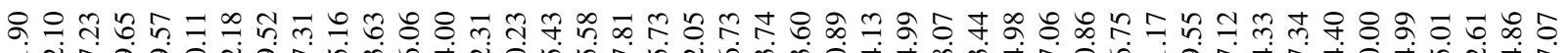

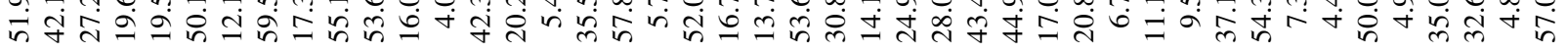

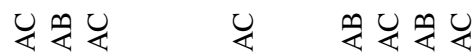

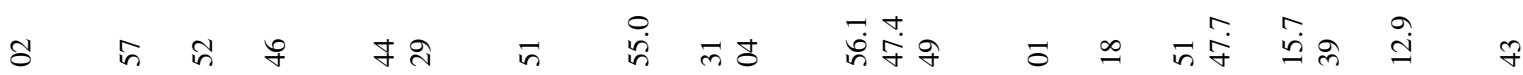

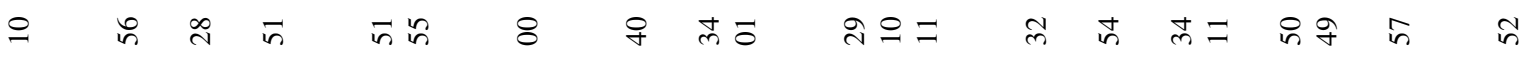

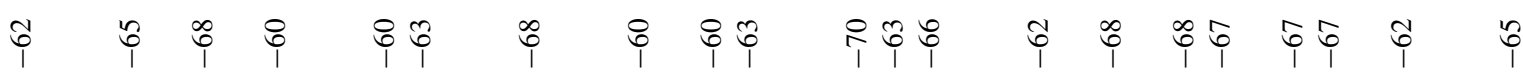

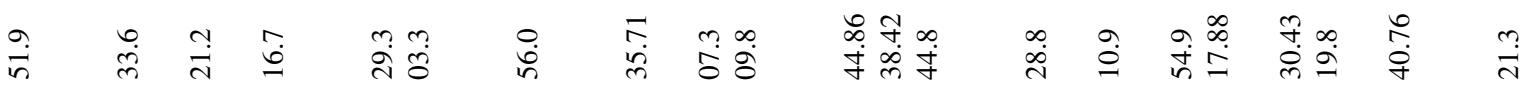

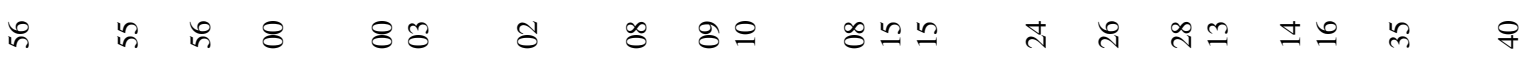

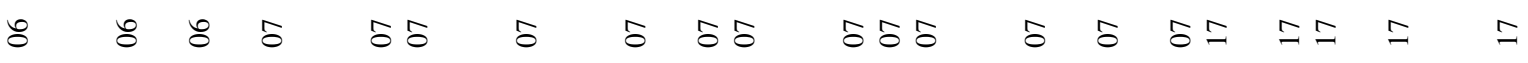

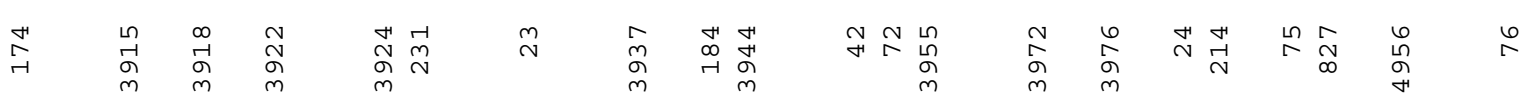

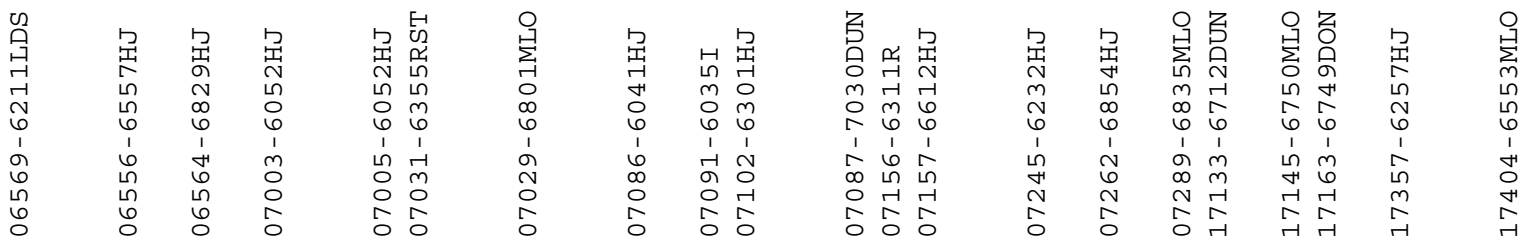




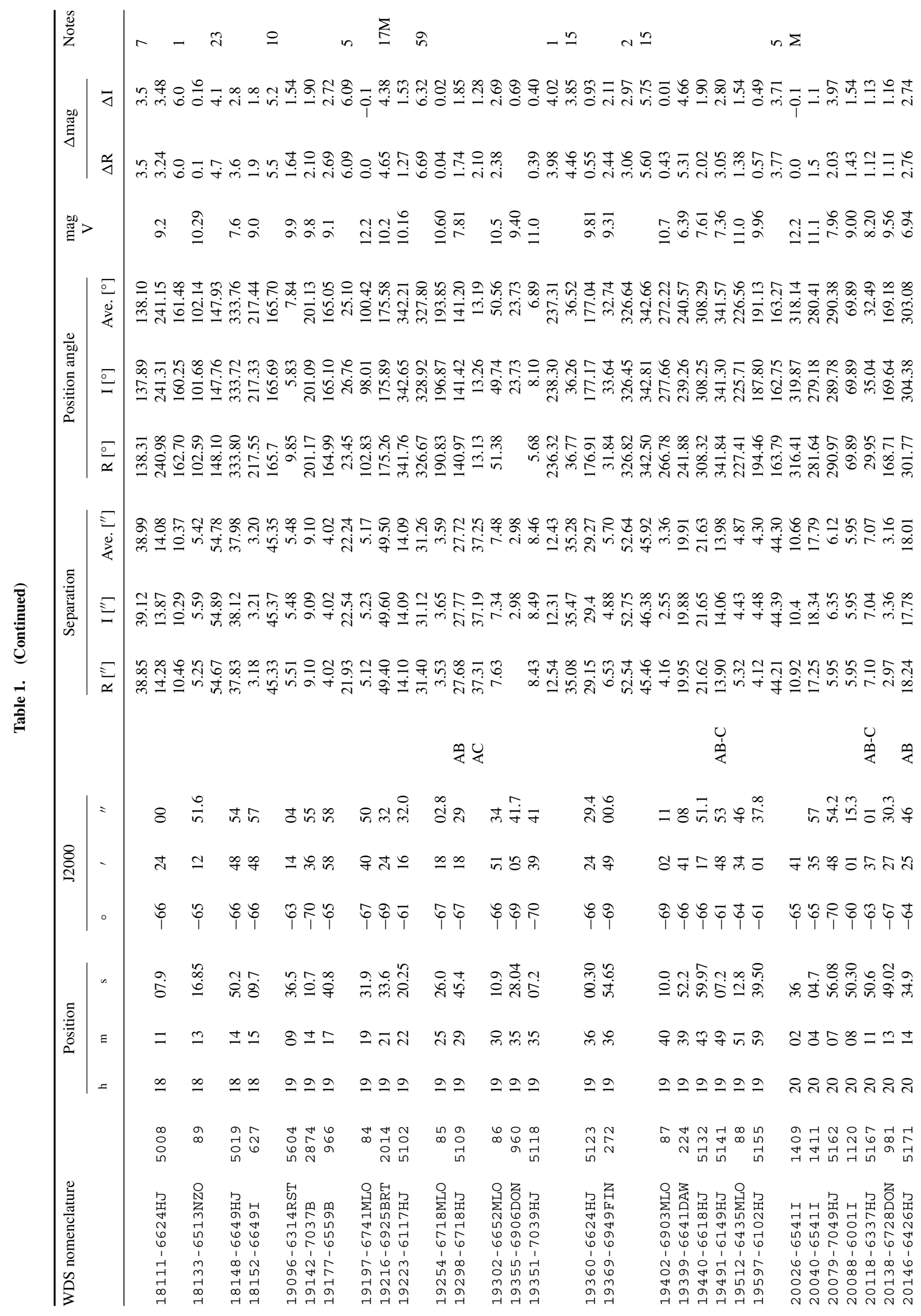




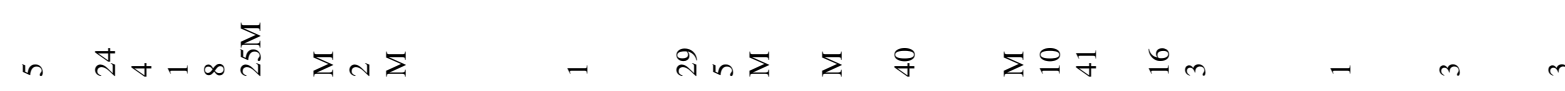

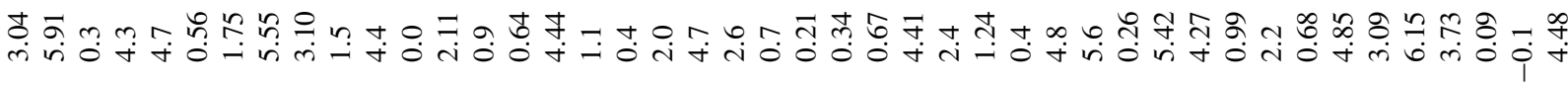

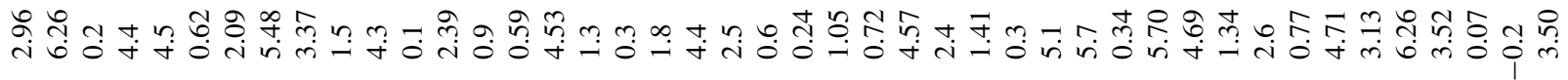

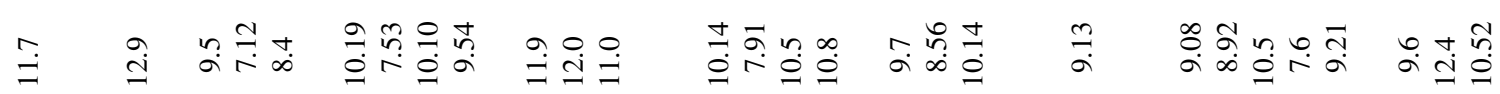
के 중

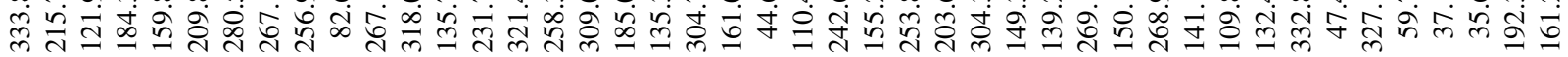

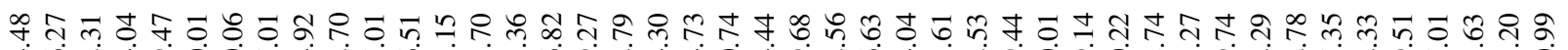

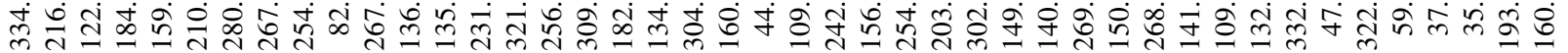

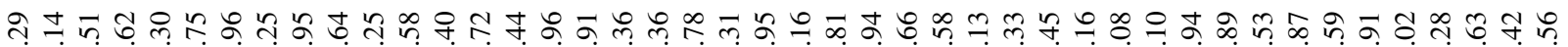

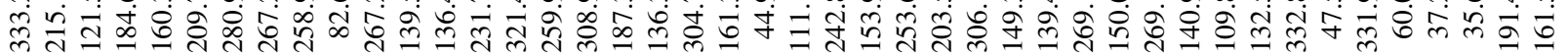

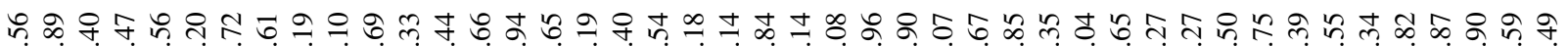

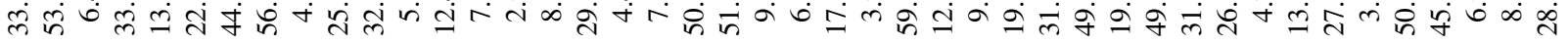

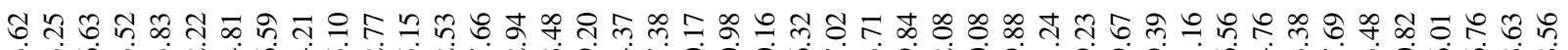
लं

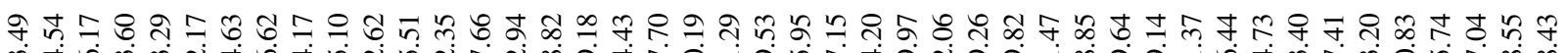
mं ¿

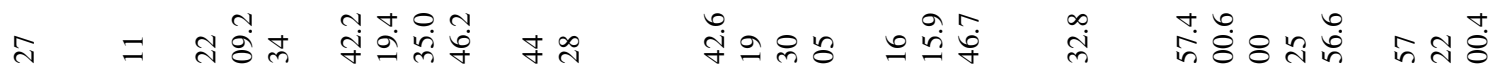

ท ำ

i 德

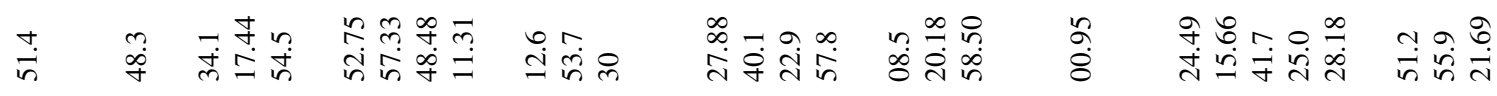

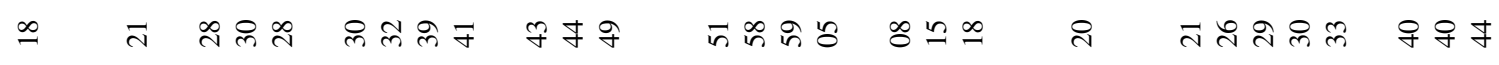

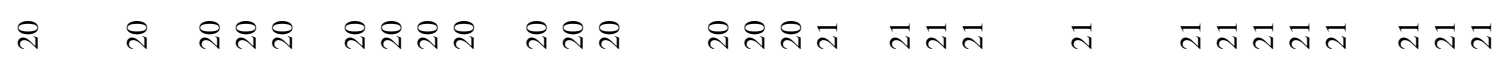

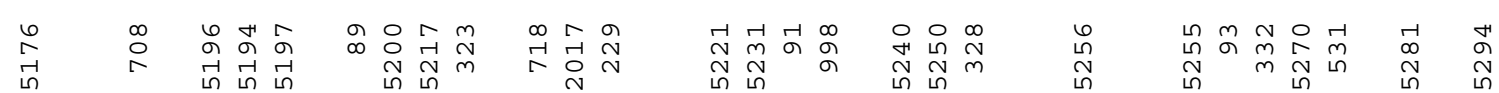

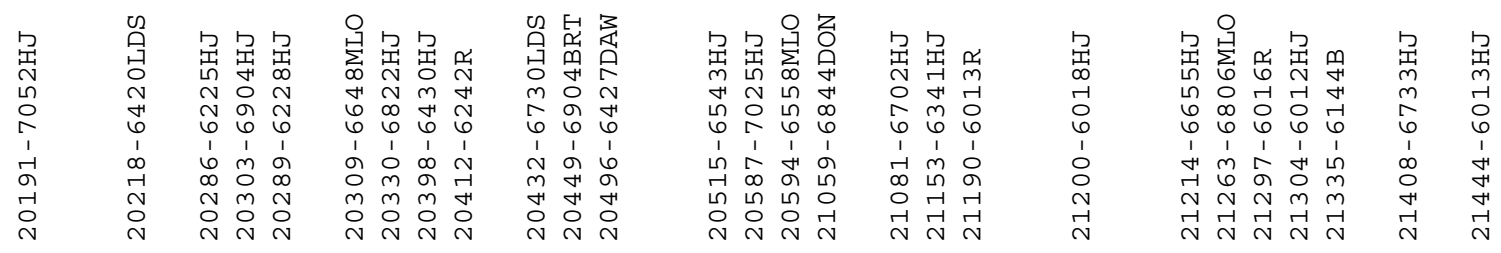




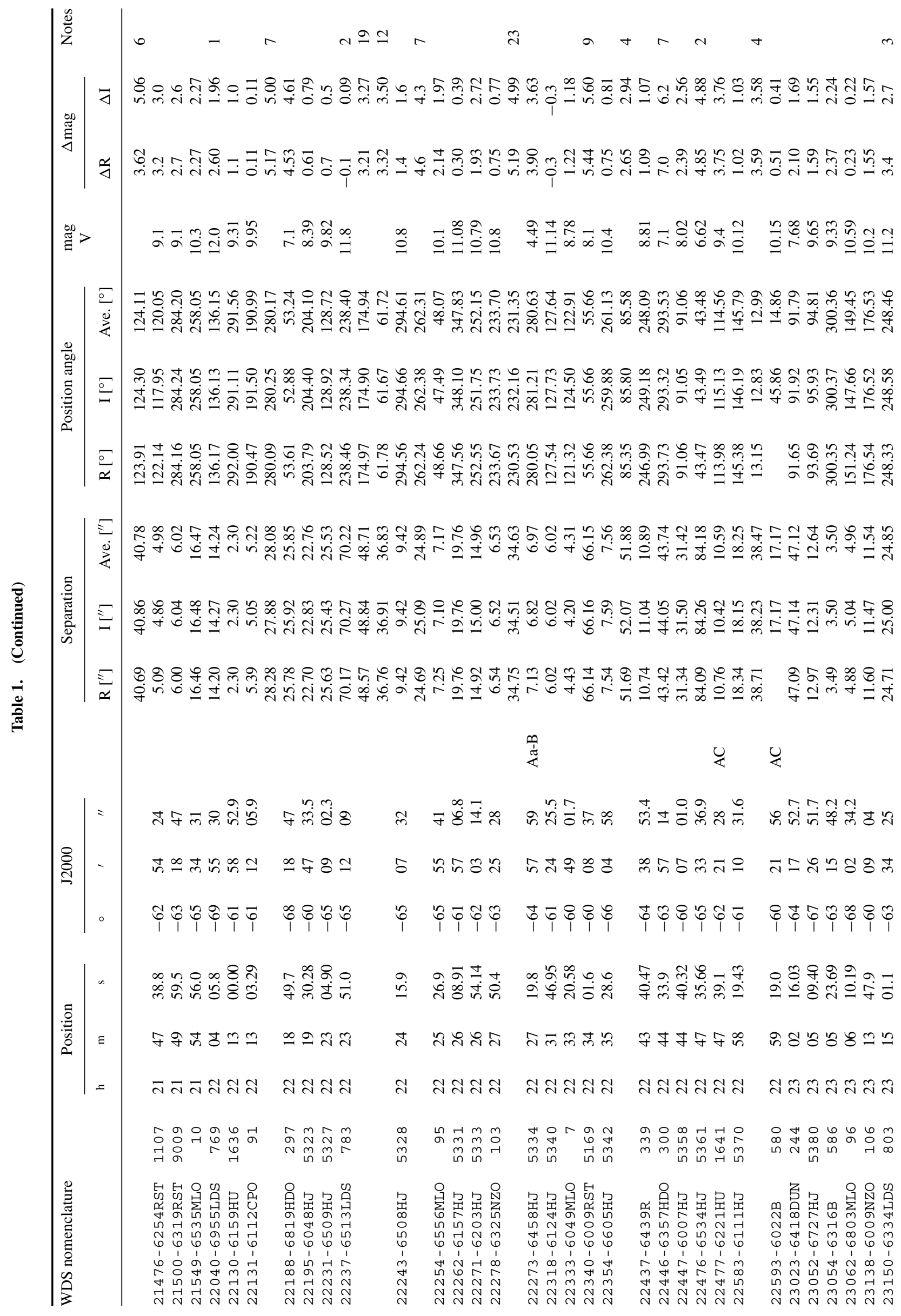


an

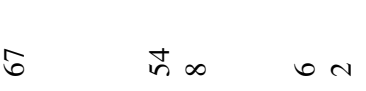

m

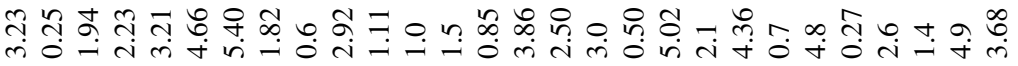

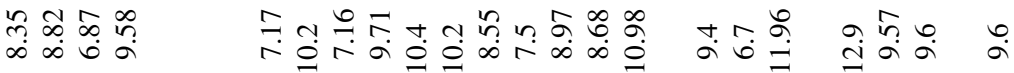

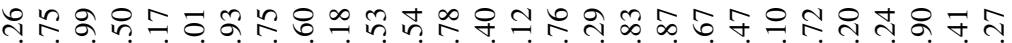

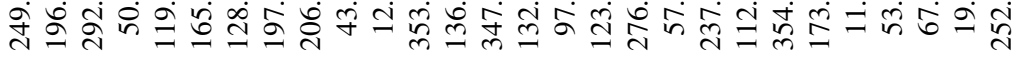

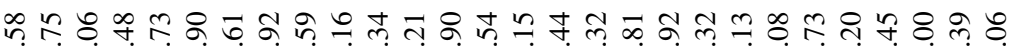

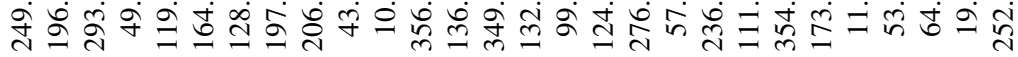

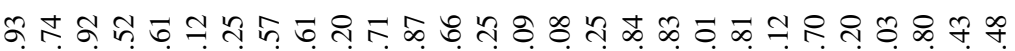

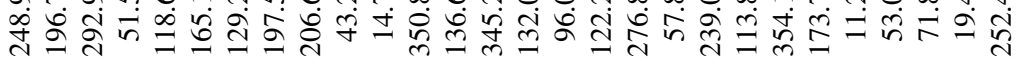

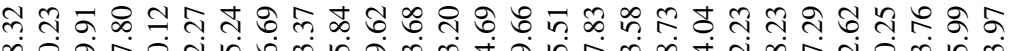

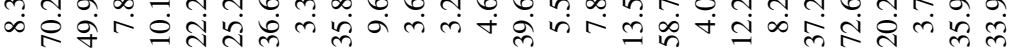

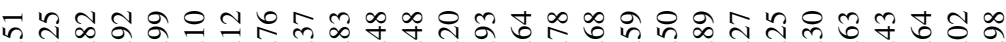

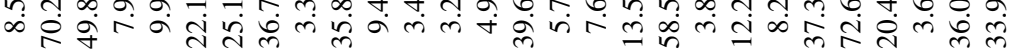

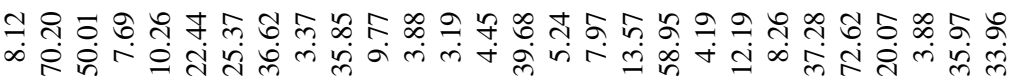

事岩

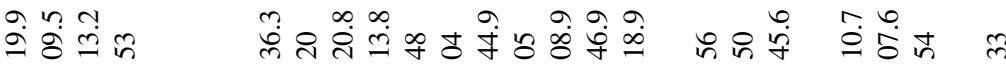

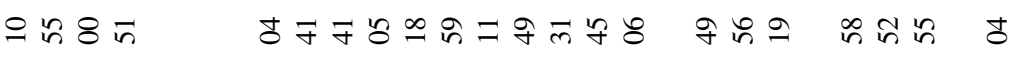

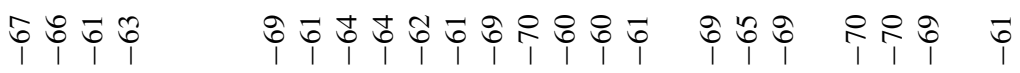

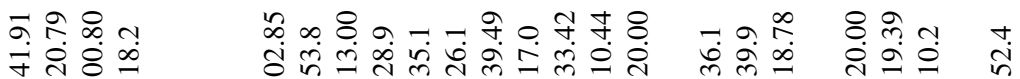

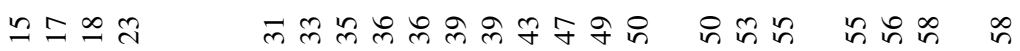

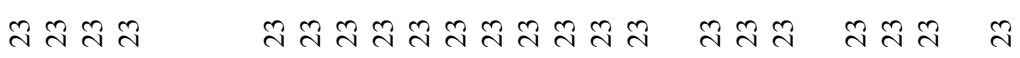

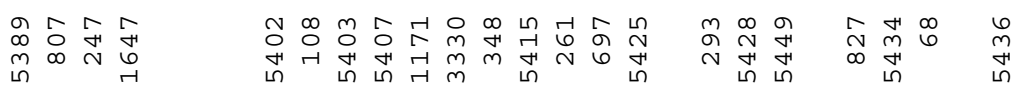

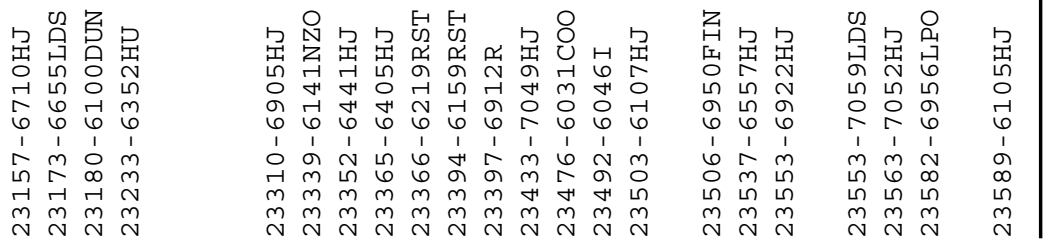




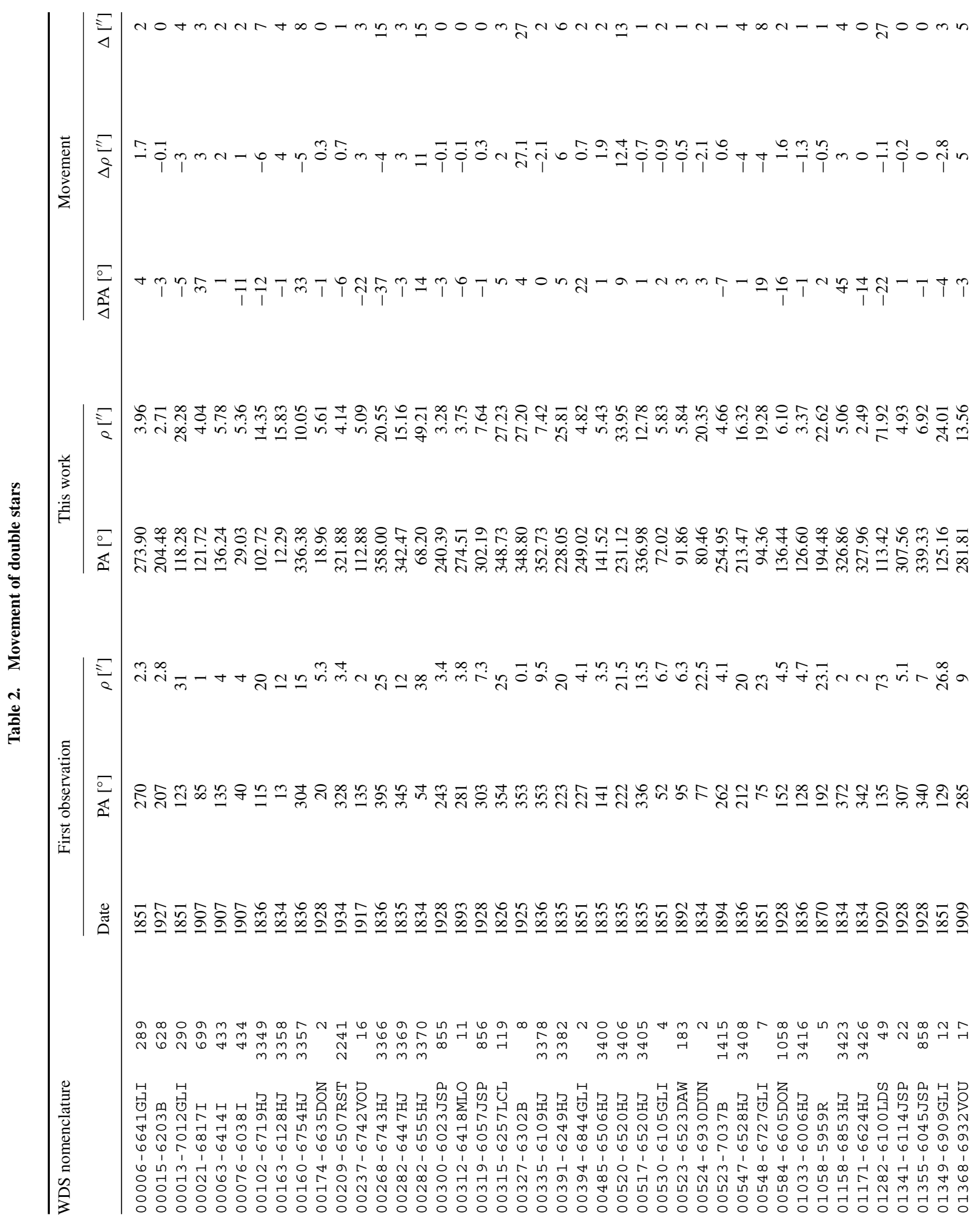




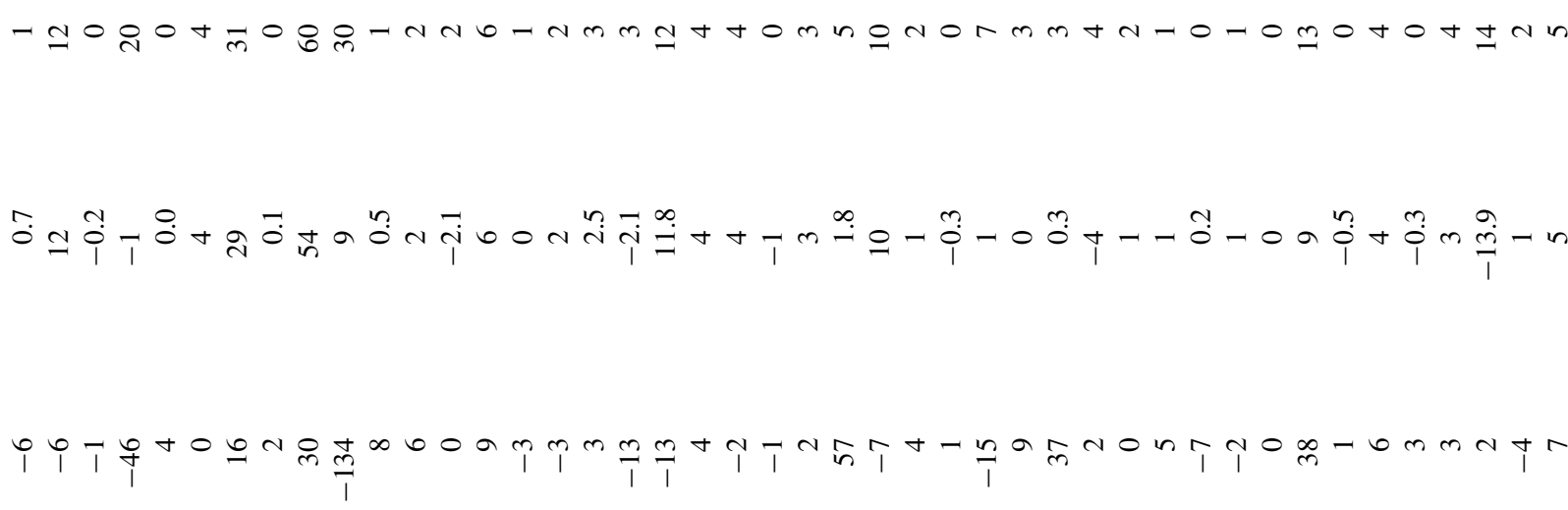

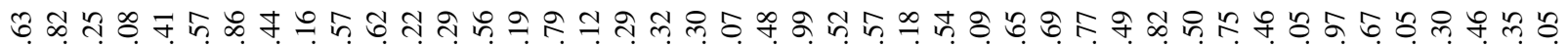
+

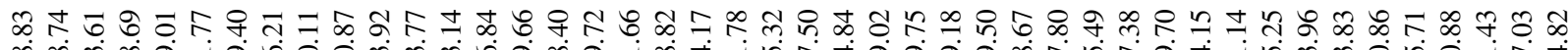
守

ri n

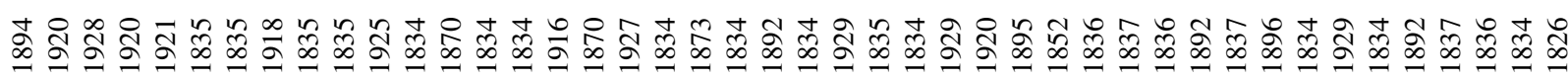

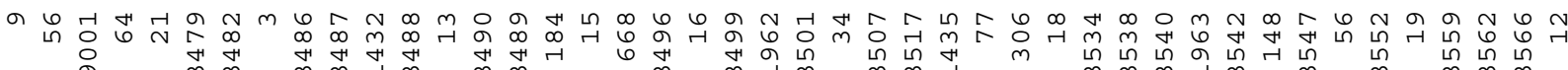

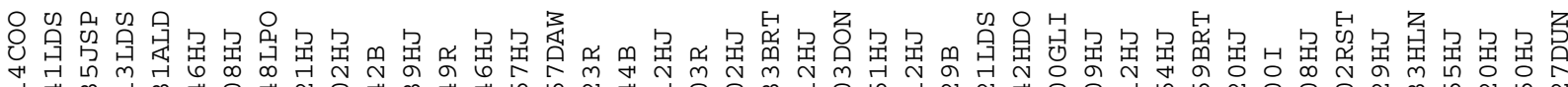

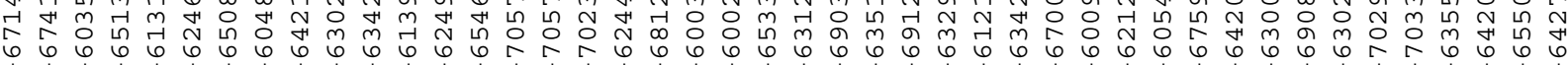

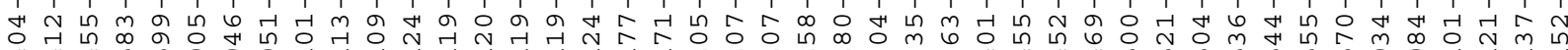

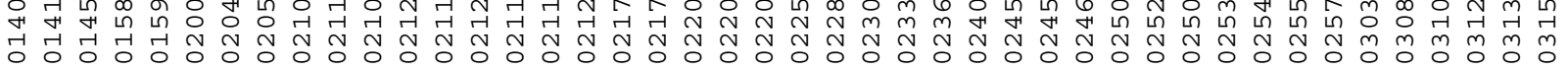




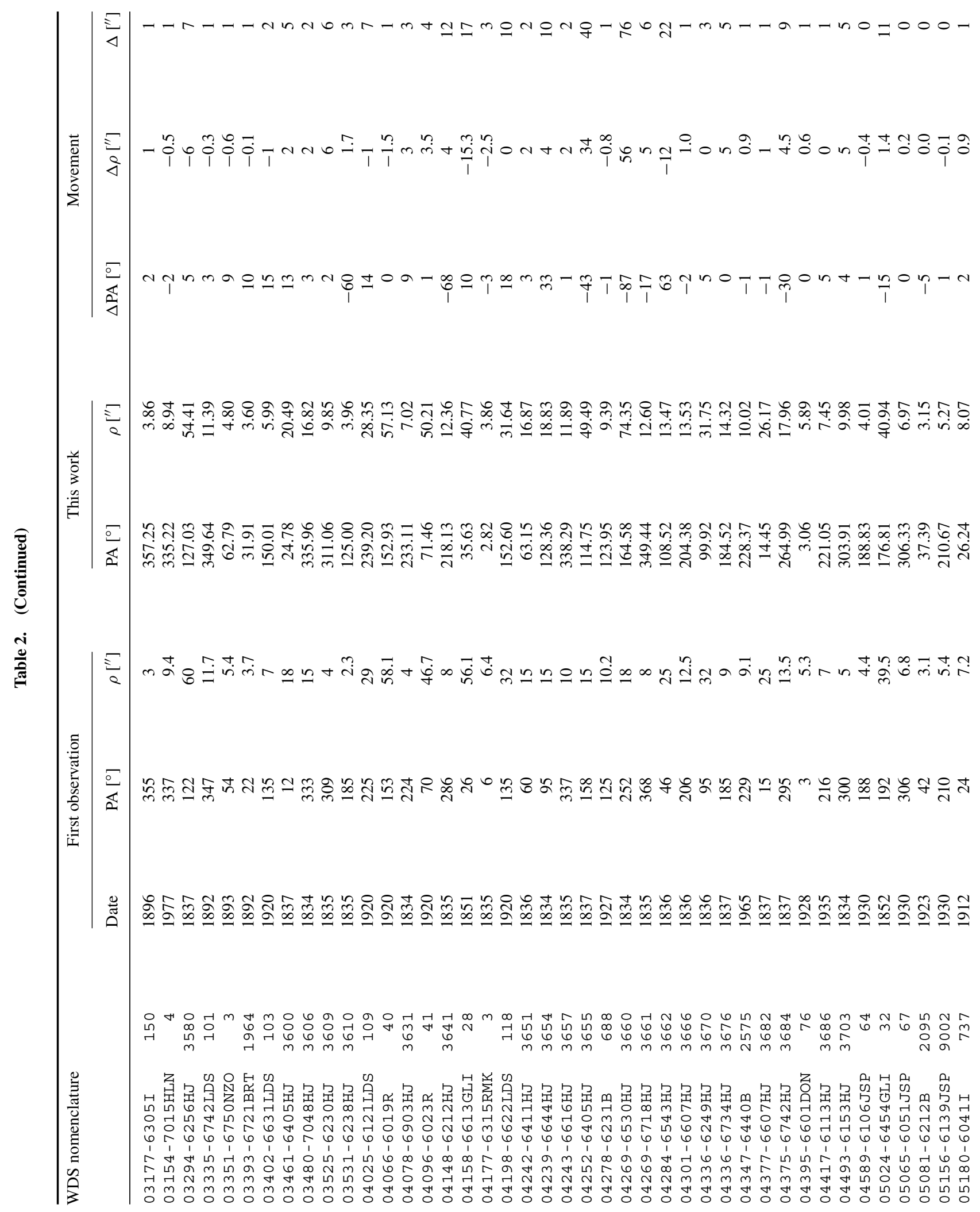




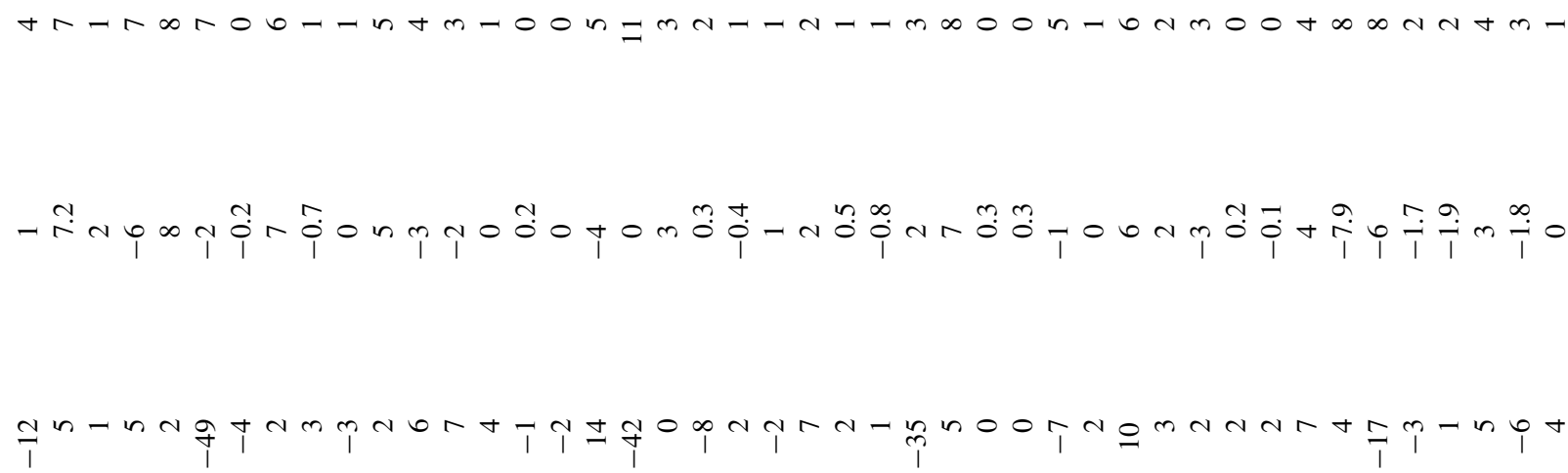

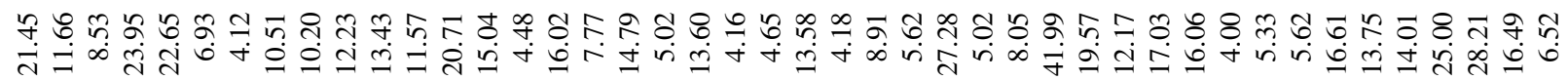

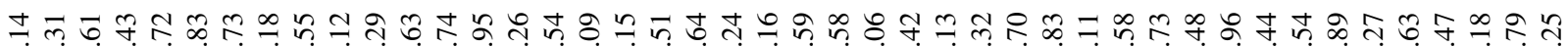

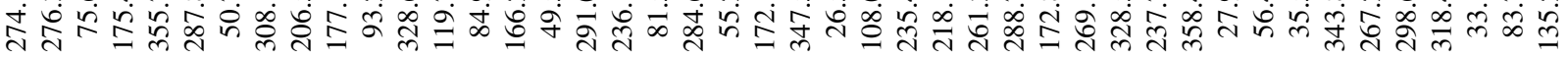

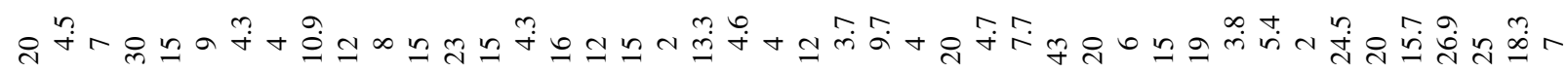

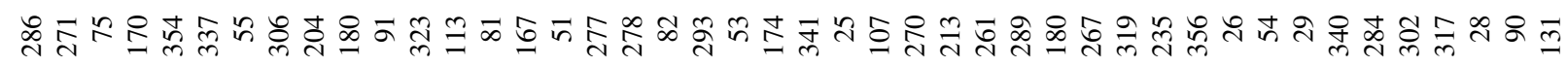

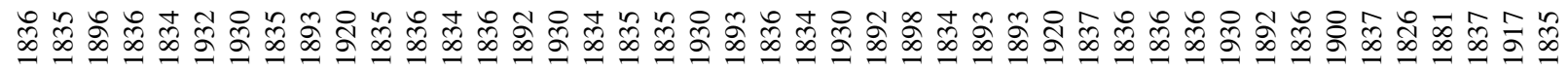

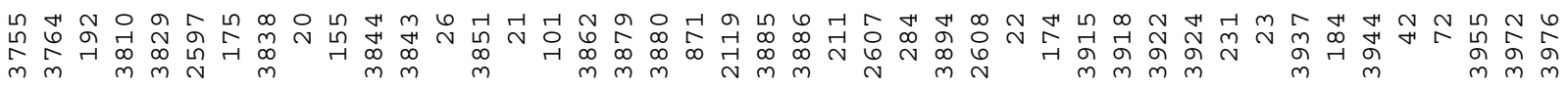

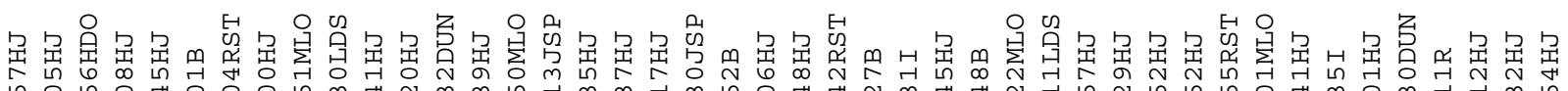

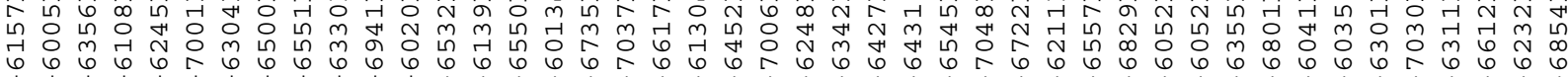

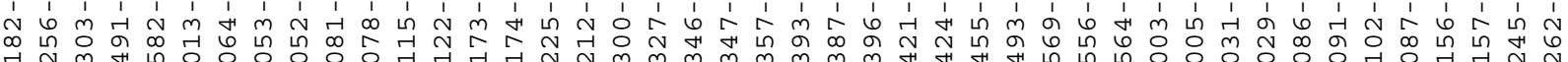

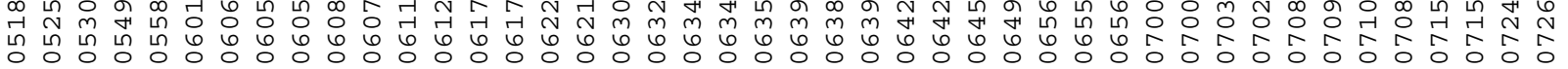




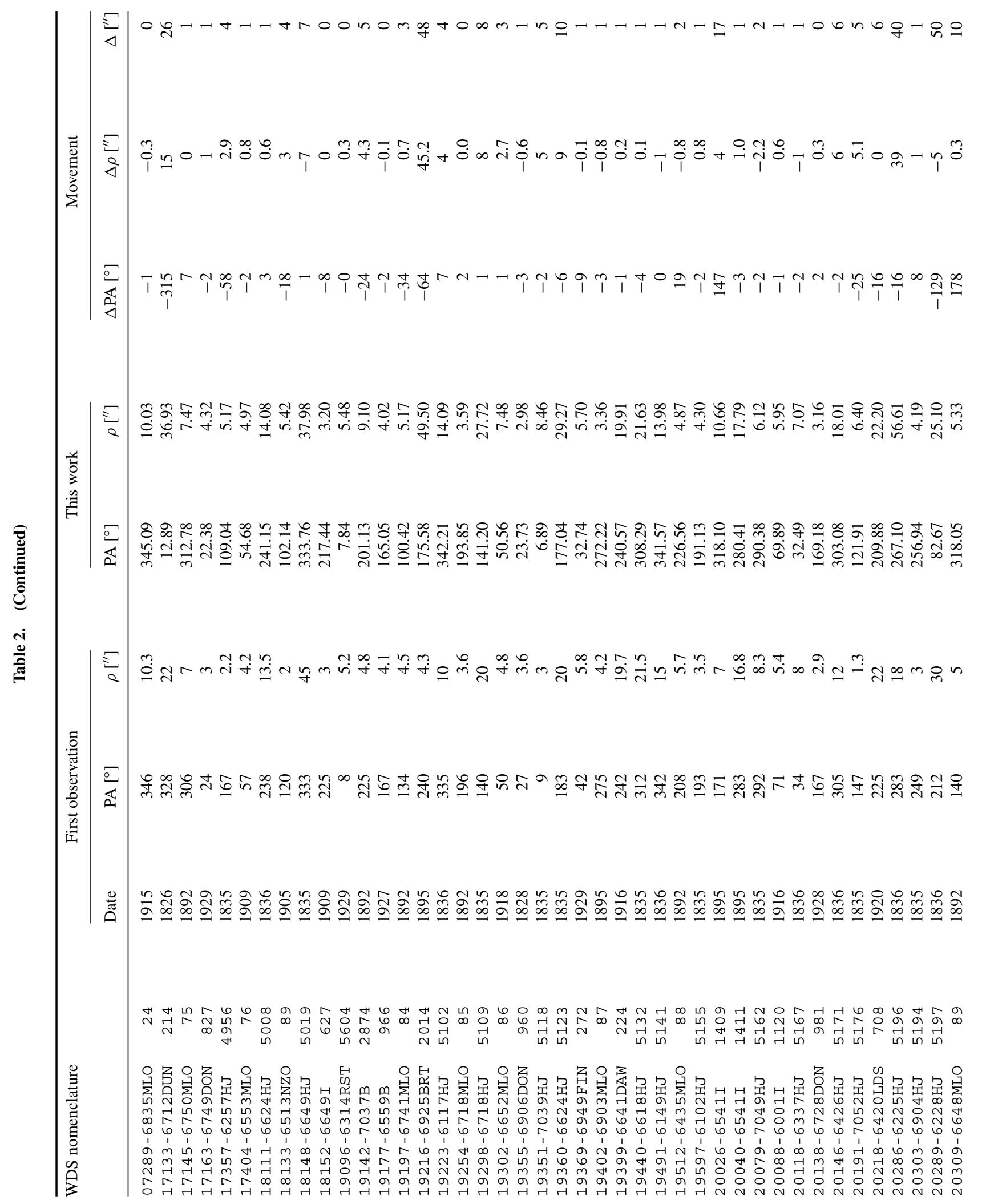




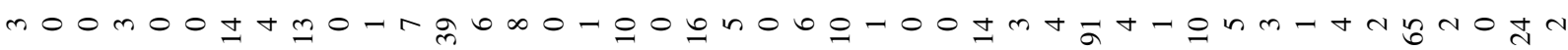

فิ TOm

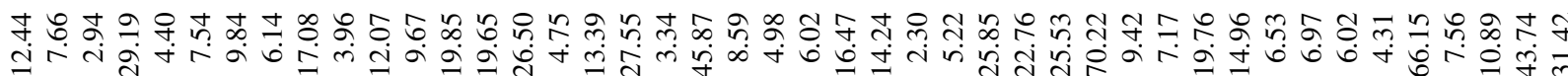

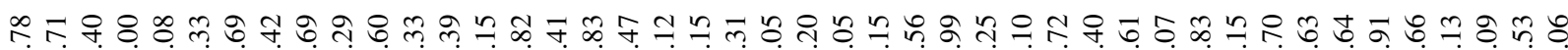

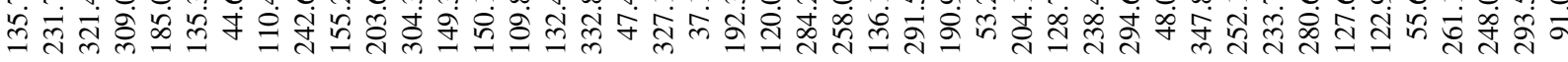

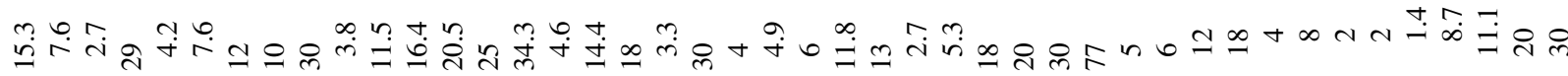

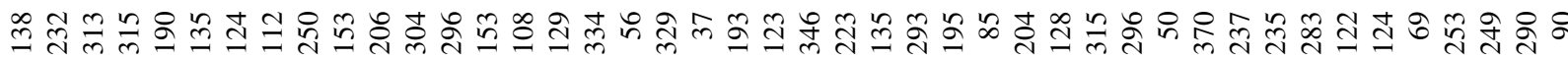

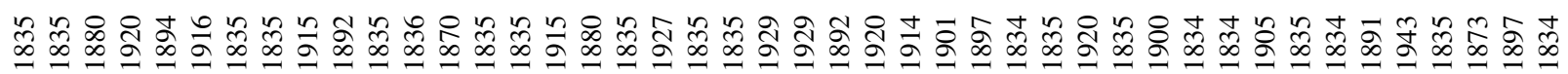

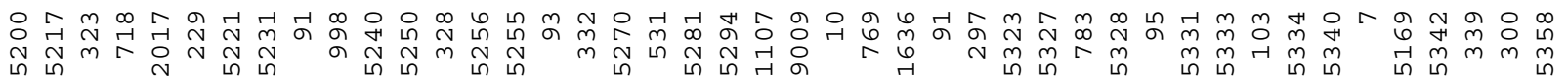

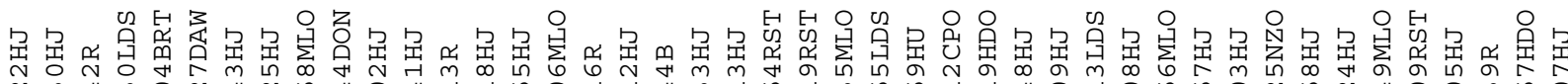

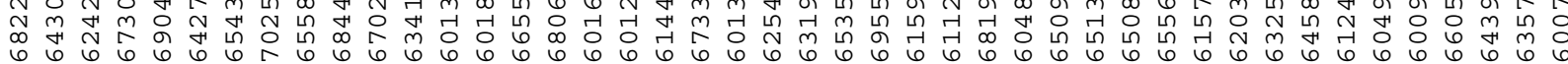

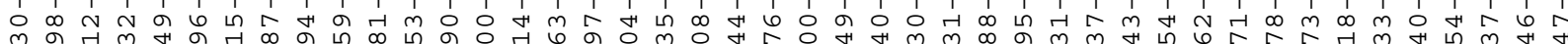

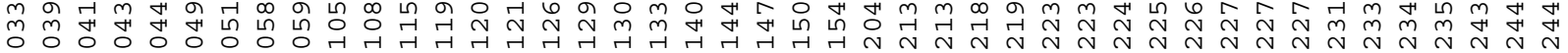




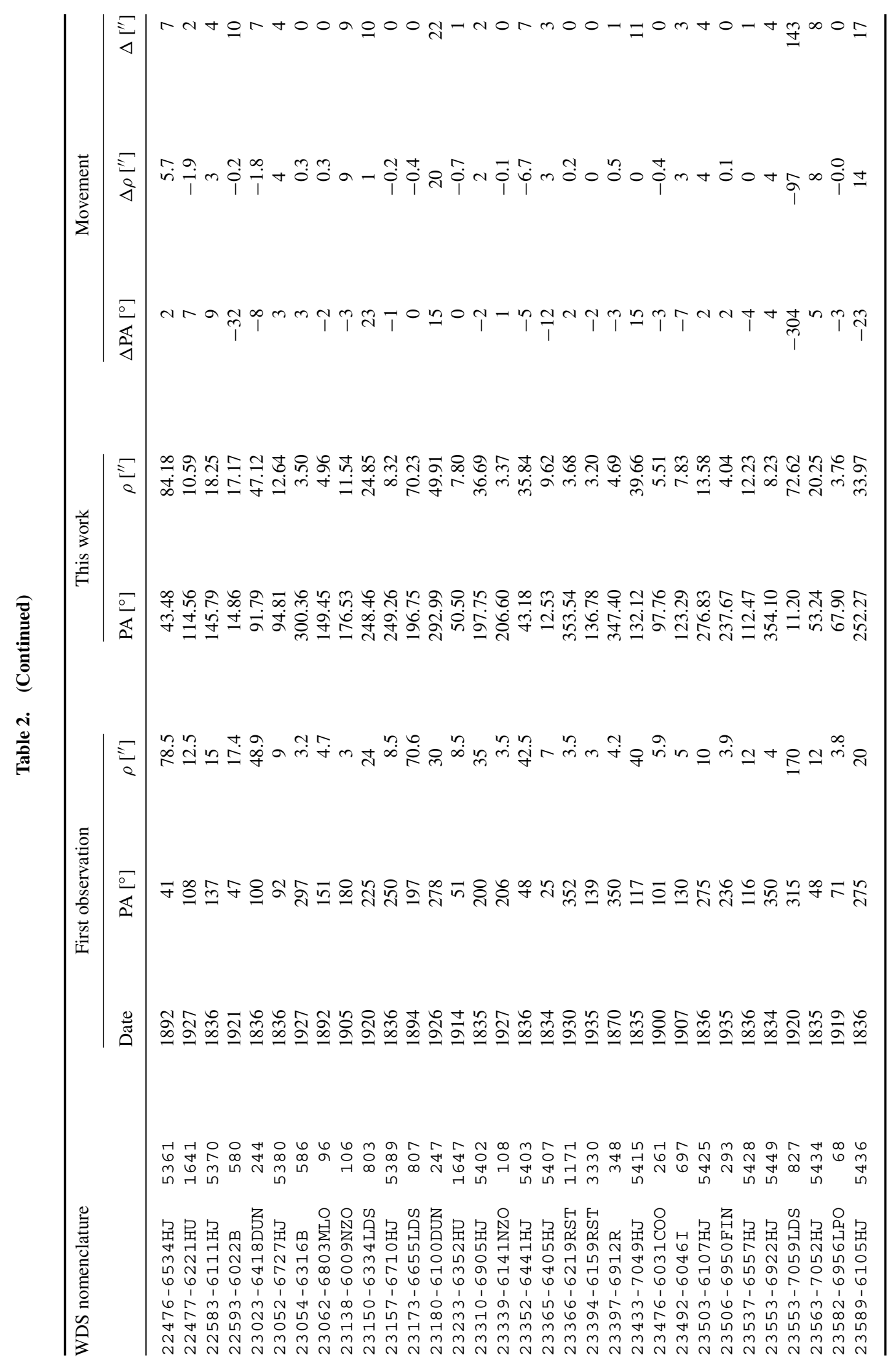


In Table 2 we present the WDS Catalog Nomenclature name of the double star, the date of the first observation as listed in the new format WDS Catalog, and the PA and $\rho$ recorded for that date. We then give the average PA and $\rho$ observed by us (epoch 1992.9). The three 'Movement' columns are $(\Delta \mathrm{PA})$ the difference in PA (ours - first), $(\Delta \rho)$ the difference in $\rho$ (ours - first) and $(\Delta)$ the movement in the period since first WDS observation; here measured as the linear distance between the first and our positions.

There are 29 secondaries (10\%) listed that have moved more than 10 arcsec over the period. Of the remainder, the distribution of movement shows that $49 \%$ percent show movement of less than 1.0 arcsec and $\sim 30 \%$ show more than 5 arcsec of motion. Given that the component of measurement uncertainty from our observations alone is $\sim 0.8$ arcsec for a pair of separation of 10 arcsec (reducing to $0.3 \operatorname{arcsec}$ at $\rho=30 \operatorname{arcsec}$ ), and that the uncertainty in the earlier observations is not known but presumed to be of the same order, it is reasonable to report that the majority of pairs listed have not moved over the period.

\section{Acknowledgements}

The authors thank the Research School of Astronomy and Astrophysics of the Australian National University for the use of their $0.6 \mathrm{~m}$ telescope and the generous donation of the R and I filters, and the University of New South Wales for their accommodation at Siding Spring Observatory. Much of this work was undertaken in the former Centre for Astronomy at the University of Western Sydney, Nepean.

\section{References}

Argue, N., et al., 1992, MNRAS, 259, 563

Bauer, C., White, G. L. \& Hons, A. H., 1994, PASA, 11, 201

Blackmore, D. R., et al., 1990, SPIE, 1237, 231

Chanamé, J. \& Gould, A., 2004, ApJ, 601, 289

Cox, A. N., 2000, Allen's Astrophysical Quantities, 4th edn (Berlin: Springer)

Dommanget, J., 1985, in The European Astrometry Satellite HIPPARCOS - Scientific Aspects of the Input Catalogue Preparation, Eds. Guyenne, T. D. \& Hunt, J. J., STIN, p. 153

Geffert, M., Sinachopoulous, D. \& Guilbert, J., 1992, in Complementary Approaches to Double and Multiple Star Research, IAU Colloquium 135, ASP Conference Series Vol. 32, Eds. McAlister, H. A. \& Hartkopf, W. I. (ASP: San Francisco), 137

Lampens, P. \& Strigachev, A., 2001, A\&A, 368, 572. doi:10.1051/ 0004-6361:20010024

Norton's Star Atlas and Reference Handbook 2000, Ed. Ridpath, I (Benjamin Cummings)

Oblak, E., et al., 1999, A\&A, 346, 523

White, G. L., et al., 1991, MNRAS, 248, 411 